\title{
Drug treatment effects on outcomes in heart failure with preserved ejection fraction: a systematic review and meta-analysis
}

\author{
Sean Lee Zheng, ${ }^{1,2,3}$ Fiona T Chan, ${ }^{3}$ Adam A Nabeebaccus, ${ }^{1,2}$ Ajay M Shah, ${ }^{1,2}$ \\ Theresa McDonagh, ${ }^{1,2}$ Darlington 0 Okonko, ${ }^{1,2}$ Salma Ayis ${ }^{4}$
}

- Additional material is published online only. To view please visit the journal online (http://dx.doi.org/10.1136/ heartjnl-2017-311652).

${ }^{1}$ Cardiovascular Division, King's College Hospital London, British Heart Foundation Centre of Research Excellence, London, UK

${ }^{2}$ Department of Cardiovascular Medicine, King's College Hospital, London, UK ${ }^{3}$ Imperial College Healthcare NHS Trust, London, UK ${ }^{4}$ Department of Primary Care and Public Health Sciences, King's College London, London, UK

\section{Correspondence to} Dr Sean Lee Zheng,

Cardiovascular Division, King's College Hospital London, British Heart Foundation Centre of Research Excellence, Imperial College Healthcare NHS Trust, SE5 9RS, London, UK; sean. zheng@nhs.net

Received 6 April 2017 Revised 19 June 2017 Accepted 5 July 2017

Published Online First 5 August 2017

Check for updates

To cite: Zheng SL, Chan FT, Nabeebaccus AA, et al. Heart 2018;104:407-415.

\section{ABSTRACT}

Background Clinical drug trials in patients with heart failure and preserved ejection fraction have failed to demonstrate improvements in mortality.

Methods We systematically searched Medline, Embase and the Cochrane Central Register of Controlled Trials for randomised controlled trials (RCT) assessing pharmacological treatments in patients with heart failure with left ventricular (LV) ejection fraction $\geq 40 \%$ from January 1996 to May 2016. The primary efficacy outcome was all-cause mortality. Secondary outcomes were cardiovascular mortality, heart failure hospitalisation, exercise capacity (6-min walk distance, exercise duration, $\mathrm{VO}_{2}$ max), quality of life and biomarkers (B-type natriuretic peptide, $\mathrm{N}$-terminal pro-B-type natriuretic peptide). Random-effects models were used to estimate pooled relative risks (RR) for the binary outcomes, and weighted mean differences for continuous outcomes, with $95 \% \mathrm{Cl}$.

Results We included data from 25 RCTs comprising data for 18101 patients. All-cause mortality was reduced with beta-blocker therapy compared with placebo (RR: $0.78,95 \% \mathrm{Cl} 0.65$ to $0.94, p=0.008)$. There was no effect seen with ACE inhibitors, aldosterone receptor blockers, mineralocorticoid receptor antagonists and other drug classes, compared with placebo. Similar results were observed for cardiovascular mortality. No single drug class reduced heart failure hospitalisation compared with placebo.

Conclusion The efficacy of treatments in patients with heart failure and an LV ejection fraction $\geq 40 \%$ differ depending on the type of therapy, with beta-blockers demonstrating reductions in all-cause and cardiovascular mortality. Further trials are warranted to confirm treatment effects of beta-blockers in this patient group.

\section{INTRODUCTION}

Heart failure with preserved left ventricular (LV) ejection fraction (HFpEF) is a heterogeneous clinical syndrome defined by the presence of signs and symptoms of heart failure without evidence of reduced LV ejection fraction (typically considered as $<40 \%) .{ }^{1}$ While significant advances have been made in the treatment of heart failure with reduced ejection fraction (HFrEF), randomised controlled trials (RCT) of pharmacological therapies in heart failure with an LV ejection fraction of $40 \%$ or more have been generally disappointing with no convincing demonstration of mortality or morbidity reduction. Updated guidelines recommend the use of diuretics for symptom relief and appropriate management of comorbidities (including hypertension), while acknowledging the absence of specific disease-modifying therapies in this condition. ${ }^{12}$

Although trial evidence demonstrating improvements in mortality have been inconsistent and largely neutral, several trials have suggested that drug therapy may improve exercise tolerance and quality of life. ${ }^{3}$ Since patients with HFpEF tend to be older with more comorbidities than their HFrEF counterparts, ${ }^{45}$ the efficacy of drug treatments might best be evaluated by their effects on hospitalisation, functional status, symptoms and quality of life. ${ }^{1}$

In this study, we aimed to systematically review the clinical trials of patients with HFpEF (defined as LV ejection fraction $\geq 40 \%$ ), and identify treatment effects on mortality, heart failure hospitalisation, functional status and biomarker levels.

\section{METHODS}

This article has been reported in accordance with the Preferred Reporting Items for Systematic Reviews and Meta-Analyses. ${ }^{6}$ No published study protocol exists for this meta-analysis.

\section{Definition of heart failure with preserved ejection fraction}

The latest European Society of Cardiology guidelines introduced the term heart failure with mid-range ejection fraction (HFmrEF), categorising an intermediate group of patients with an LV ejection fraction of between $40 \%$ and $49 \%$, with HFpEF defined as an LV ejection fraction $\geq 50 \%$ with the same echocardiographic criteria. ${ }^{1}$ The American College of Cardiology defines HFpEF as an LV ejection fraction $>40 \%$, with anything from $41 \%$ to $49 \%$ as borderline HFpEF. ${ }^{2}$ While the terminology has changed in the process of this meta-analysis being undertaken, the aim of this study was to identify treatment effects in the group of patients with heart failure with LV ejection fraction $\geq 40 \%$, for which no guideline-recommended therapies currently exist. In the HFpEF population, RCTs have used various LV ejection fraction cut-offs, ranging from $40 \%$ to $50 \%$, and therefore data summarised in this meta-analysis will include patients in the mid-range and borderline group. 
Heart failure with LV ejection fraction $\geq 40 \%$ will henceforth be referred to as HFpEF.

\section{Search strategy and selection criteria}

A systematic search of Medline, Embase and the Cochrane Central Register of Controlled Trials was performed using the search strategy documented in the online supplementary materials. Results were filtered for randomised controlled trials using predesigned and validated filters. The search was run on 1 May 2016, with results included from database inception to 1 May 2016. The search was rerun on 1 April 2017 and no additional articles were identified. The reference lists of included studies were searched for additional analyses. A systematic approach was used to identify systematic reviews and meta-analyses published during this period, which were hand-screened for additional trials.

Trials were considered eligible if they were (a) RCT; (b) enrolled participants with heart failure and documented LV ejection fraction $\geq 40 \%$; (c) compared drug therapy with placebo, no treatment, diuretic treatment or standard medical treatment, with a minimum follow-up of at least 12 weeks and (d) provided information on prespecified primary and secondary end points that included all-cause mortality, cardiovascular mortality, heart failure hospitalisation, exercise capacity (6 min walk distance (6MWD), exercise duration, $\mathrm{VO}_{2}$ max), quality of life as measured using the Minnesota Living With Heart Failure Questionnaire (MLHFQ) and biomarkers (B-type natriuretic peptide (BNP), N-terminal pro-B-type natriuretic peptide (NT-proBNP)). Non-English language publications were excluded. We allowed secondary publications of included trials if they reported additional outcomes that were not present in the original article.

After removal of duplicates, the title and abstracts of initial search results were screened for relevance. The full texts of remaining results were independently assessed by two authors (SLZ, FTC) for inclusion based on predetermined inclusion and exclusion criteria. The final list of included studies was decided by discussion between authors and required full agreement. No disagreements required resolution by a third reviewer. Study selection flow diagram is shown in figure 1.

\section{Data extraction and quality assessment}

Data were extracted using piloted forms, independently and in duplicate by two authors (SLZ, FTC), and were transcribed

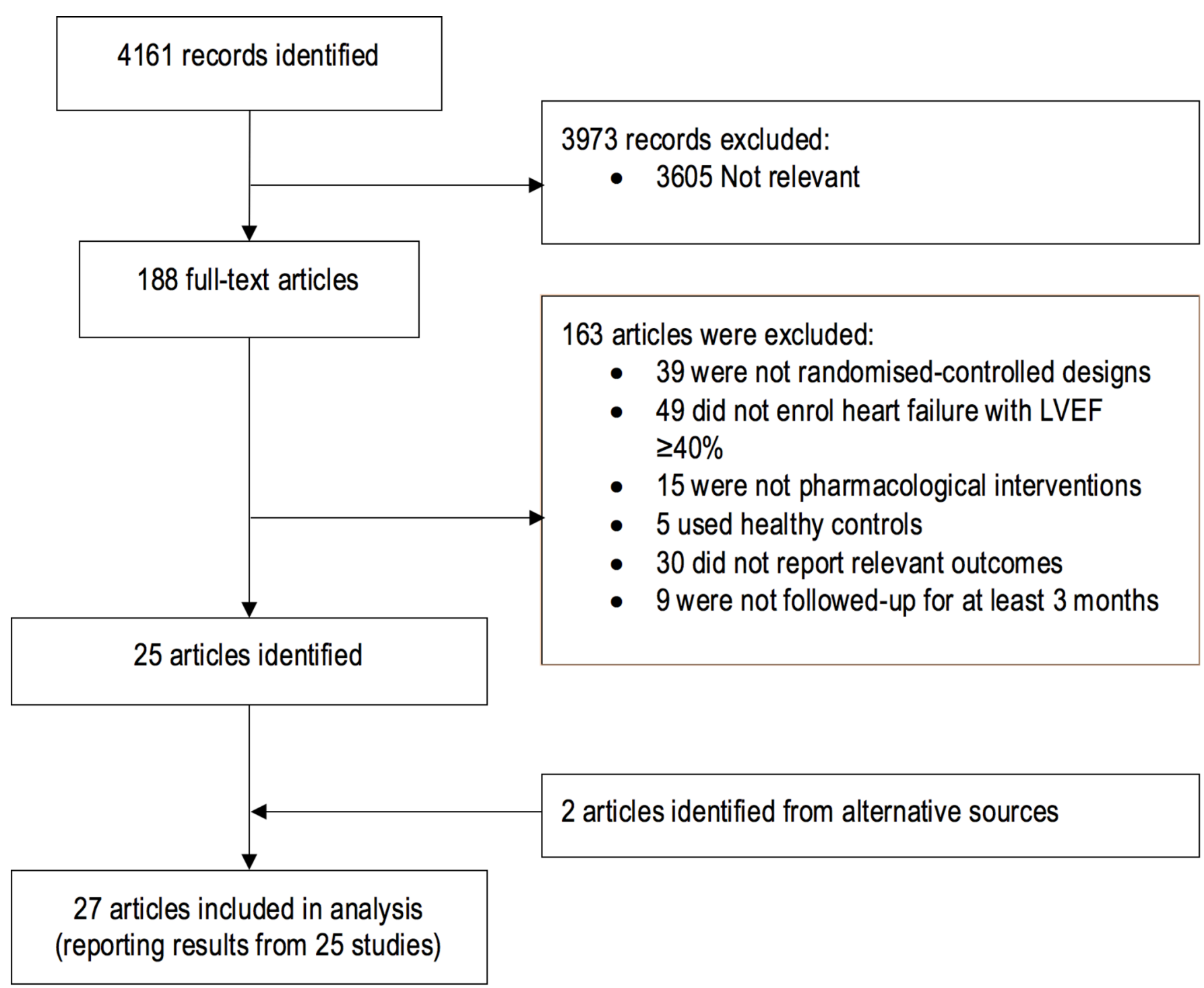

Figure 1 Study flow diagram of the trial selection process. LVEF, left ventricular ejection fraction. 
onto a dedicated database. The data extracted from each report included baseline patient characteristics (age, sex, comorbidities, mean ejection fraction), study inclusion criteria, study drug and control treatment, follow-up duration and end point data.

The Cochrane Collaboration risk of bias tool was used to assess risk of bias. Disagreements in abstracted data were adjudicated by a third reviewer (AAN). Egger test was used to identify asymmetry of funnel plots for publication bias.

\section{Statistical analysis}

Random-effects models were used to estimate pooled effect size from aggregate data. ${ }^{7}$ For binary outcomes, estimates were summarised as relative risk (RR) and 95\% CI. For continuous outcomes, weighted mean difference (MD) and 95\% CIs were calculated. Studies with no outcome events in the treatment or placebo groups, and therefore did not contribute to the risk ratios, were excluded from the RR pooled estimates, and were summarised using risk differences. Estimates and 95\% CIs were graphically presented using Forest plots. ${ }^{8} \chi^{2}$ test was used to compare differences between stratified subgroup RR.

Results were stratified by drug type (beta-blocker, ACE inhibitor, angiotensin receptor blocker (ARB), mineralocorticoid receptor antagonist (MRA), combined renin-angiotensin-aldosterone system (RAAS) antagonists and other drug types), duration of follow-up (3-12 months and $>12$ months), LV ejection fraction entry threshold $(40 \%-49 \%$ and $\geq 50 \%)$ and mean LV ejection fraction $(<60 \%$ and $\geq 60 \%)$. The original protocol did not specify stratifying by patient's LV ejection fraction $(40 \%-$ $49 \%$ and $\geq 50 \%)$. No trials enrolled patients exclusively with LV ejection fraction between $40 \%$ and $49 \%$, and no trials reported subgroup analyses of this group.

Between-study heterogeneity was assessed using $\mathrm{I}^{2}$ statistics, which describes the percentage of variation across studies that is due to heterogeneity rather than chance. ${ }^{9}$ Publication bias was assessed if the number of trials was reasonable ( 10 or more) using funnel plots. No assessment was made for smaller number of trials due to the established poor performance of the test. ${ }^{10}$ Statistical analysis was performed using the software STATA V.14 and Review Manager V.5.3.

\section{RESULTS}

The electronic search identified 4161 articles that were screened and evaluated for eligibility based on title and abstract only (figure 1). After removal of duplicate (368) and non-relevant (3605) articles, 188 articles were evaluated in full-text for eligibility. A further 163 articles were excluded, and two additional eligible articles were identified from searching systematic reviews and reference lists. In all, 27 articles were included for meta-analysis, comprising data from 25 trials, with 28 separate comparisons (see online supplementary file 1). In total, 18101 patients were randomised to either drug intervention or placebo, control or standard medical therapy. There were six betablocker trials enrolling 1299 patients, 5 ACE inhibitor (1305 participants), 6 ARB (9704 participants) and 5 MRA (4003 participants) trials. Other drugs tested include one digoxin (988 participants), two calcium channel blocker (242 participants), one sildenafil (216 participants), one sitaxsentan (192 participants) and one doxazosin (145 participants) trial. Seven studies used an LV ejection fraction threshold of $40 \%$, and nine used LV ejection fraction thresholds of $45 \%$ and $50 \%$. The inclusion criteria for Study of Effects of Nebivolol Intervention on Outcomes and Rehospitalization in Seniors With Heart Failure (SENIORS) included either an LV ejection fraction threshold of $35 \%$ or previous hospitalisation for heart failure regardless of ejection fraction, with subgroup analysis of patients with LV ejection fraction $\geq 40 \%$, and so the study was included in the pooled analysis. ${ }^{11}$

\section{Effect of therapy on all-cause mortality, cardiovascular mortality and heart failure hospitalisation Stratification by drug class}

The search identified 16 studies that reported all-cause mortality events in one or both arms, which were pooled for analysis. Beta-blockers were the only pharmacological agent that reduced the risk of all-cause mortality compared with control (RR: $0.78,95 \% \mathrm{CI}: 0.65$ to 0.94 , $\mathrm{p}=0.008, \mathrm{n}=1046)^{11-13}$ (figure 2, table 1). Pooled analysis of ACE inhibitor (RR: $1.10,95 \%$ CI: 0.85 to $1.43, \mathrm{p}=0.46$, $\mathrm{n}=1234),{ }^{14-17}$ ARB (RR: $1.02,95 \%$ CI: 0.93 to $1.12, \mathrm{p}=0.71$, $\mathrm{n}=7257)^{14} 18-20$ and MRA (RR: $0.92,95 \%$ CI: 0.79 to 1.08 , $\mathrm{p}=0.32, \mathrm{n}=3867)^{21} 22$ trials showed no effect on all-cause mortality compared with control. Pooled trials of drugs blocking the renin-angiotensin-aldosterone system (RAAS) (ACE inhibitor, $\mathrm{ARB}$ and MRA) did not reduce all-cause mortality (RR: $1.00,95 \%$ CI 0.93 to $1.08, \mathrm{p}=0.97, \mathrm{n}=12358$ ) (see online supplementary figure 1A). Pooled effects of other drug types showed no difference (RR: $0.95,95 \% \mathrm{CI}: 078$ to $1.15, \mathrm{p}=0.58, \mathrm{n}=1576){ }^{162324}$

Cardiovascular mortality was reduced in beta-blocker therapy compared with controls (RR: $0.75,95 \% \mathrm{CI}$ : 0.60 to 0.94 , $\mathrm{p}=0.01, \mathrm{n}=1046$ ), whereas ACE inhibitors (RR: $0.94,95 \% \mathrm{CI}$ : 0.62 to $1.43, \mathrm{p}=0.77, \mathrm{n}=945)$ and $\mathrm{ARB}(\mathrm{RR}: 1.02,95 \% \mathrm{CI}$ : 0.90 to $1.14, p=0.79, n=7257)$ had no effect on cardiovascular mortality (figure 3 ). There was no effect of pooled RAAS blockade (RR $0.99,95 \%$ CI 0.89 to $1.09, p=0.77$ ) on cardiovascular mortality compared with controls (see online supplementary figure $2 \mathrm{~A}$ ).

Pooled RAAS blockade reduced the risk for heart failure hospitalisation $(\mathrm{RR}=0.90,95 \% \mathrm{CI}: 0.82$ to $0.98, \mathrm{p}=0.01, \mathrm{n}=11765)$, though ACE inhibitors (RR: 0.86, 95\% CI: 0.64 to 1.15 , $\mathrm{p}=0.32, \mathrm{n}=1019)$ and ARB (RR 0.92, 95\% CI: 0.83 to 1.02 , $\mathrm{p}=0.13, \mathrm{n}=7301$ ) had no effect individually (online supplementary figure $3 \mathrm{~A}$ and figure 4). There was no effect of beta-blocker therapy on heart failure hospitalisation (RR: $0.67,95 \%$ CI: 0.42 to $1.07, \mathrm{p}=0.10, \mathrm{n}=382$ ) (figure 4). Only one trial of MRA (Treatment of Preserved Cardiac Function Heart Failure With an Aldosterone Antagonist trial (TOPCAT) ${ }^{21}$ reported cardiovascular mortality and heart failure hospitalisation outcomes (HR: $0.90, p=0.35$ and HR: $0.83, p=0.04$, respectively).

Meta-analysis of all trials revealed that pharmacotherapy did not improve all-cause (RR: $0.96,95 \%$ CI: 0.90 to 1.03 , $\mathrm{p}=0.26$ ) or cardiovascular (RR: $0.95,95 \% \mathrm{CI}: 0.87$ to 1.03 , $\mathrm{p}=0.23)$ mortality, but reduced heart failure hospitalisation (RR: $0.88,95 \%$ CI: 0.81 to $0.95, p=0.002$ ). When all trials (including trials with no events in both arms) were considered using the mean weighted absolute risk difference, no significant difference was observed between treatment and control groups for all-cause mortality (Risk Difference (RD): $-0.00 \%$, 95\% CI: $-0.01 \%$ to $0.01 \%, \mathrm{p}=0.99, \mathrm{n}=15340$ ), or cardiovascular mortality (RD: $-0.00 \%, 95 \% \mathrm{CI}:-0.01 \%$ to $0.01 \%$, $\mathrm{p}=0.70, \mathrm{n}=14257$ ) (see online supplementary figure $1 \mathrm{~B}$ and 2B). Heart failure hospitalisation showed a small but significant difference in risk favouring treatment (RD: $-0.02 \%, 95 \% \mathrm{CI}$ : -0.03 to $-0.00 \%, \mathrm{p}=0.005, \mathrm{n}=13491)$, driven by the treatment effect of RAAS blockade (see online supplementary figure $3 \mathrm{~B}$ ). Summary of effects for all primary and secondary 


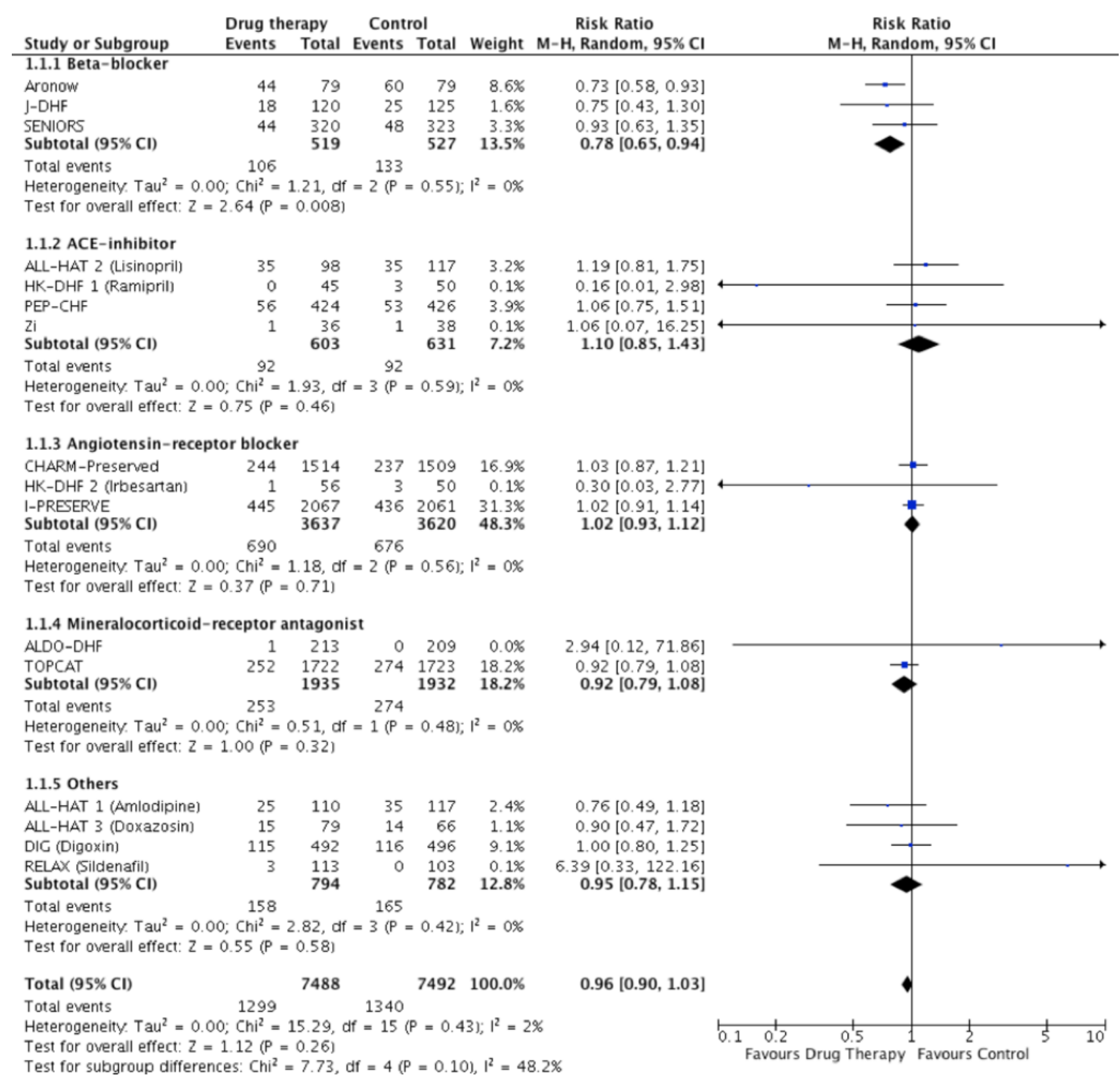

Figure 2 Pooled and individual estimates of relative risk (RR) and $95 \% \mathrm{Cl}$ of the primary outcome all-cause mortality for different therapies. Data are shown stratified by individual drug classes (beta-blockers, ACE inhibitors, angiotensin receptor blockers, mineralocorticoid receptor antagonists and other drug classes). Random-effects model used.

outcomes by stratification is shown in online supplementary table 2 .

\section{Stratification by LV ejection fraction inclusion threshold}

When analyses were stratified by LV ejection fraction inclusion threshold (40\%-49\%, $\geq 50 \%)$, neither group demonstrated significant differences in all-cause mortality, with no difference between subgroups $\left(\chi^{2}=0.05, p=0.83\right)$ (see online supplementary figure 1C). Hospitalisation for heart failure was reduced in studies that used LV ejection fraction inclusion cut-offs of $40 \%-49 \%$ (RR: $0.88,95 \% \mathrm{CI}: 0.82$ to $0.96, \mathrm{p}=0.002$ ), but not in studies using cut-offs of $50 \%$ or more (RR: $0.51,95 \% \mathrm{CI}$ : 0.18 to $1.48, \mathrm{p}=0.22)\left(\chi^{2}=1.01, \mathrm{p}=0.31\right)$. Only one study that reported cardiovascular mortality used an LV ejection fraction inclusion cut-off of $50 \%$ making a similar comparison not possible for this outcome.
Stratification by mean LV ejection fraction

Comparison of trials with a mean LV ejection fraction $<60 \%$ (five trials, $\mathrm{n}=7688$ ) with $\geq 60 \%$ (seven trials, $\mathrm{n}=6062$ ) demonstrated that neither subgroup reached statistical significance for all-cause or cardiovascular mortality, with no difference between the subgroups $\left(\chi^{2}=1.05, p=0.31\right)$ (online supplementary figures $1 \mathrm{E}$ and $2 \mathrm{E}$, table 1$)$. Heart failure hospitalisation was reduced in studies with a mean LV ejection fraction $<60 \%$ (RR: 0.85 , 95\% CI 0.76 to $0.94, \mathrm{p}=0.002)$, but not in studies with higher mean LV ejection fractions (RR: $0.92,95 \% \mathrm{CI}: 0.82$ to 1.04 , $\mathrm{p}=0.19)\left(\chi^{2}=1.03, \mathrm{p}=0.31\right)$.

Stratification by follow-up duration

Trials with shorter follow-up (3-12 months) demonstrated statistically significant reductions in relative risk for both mortality 


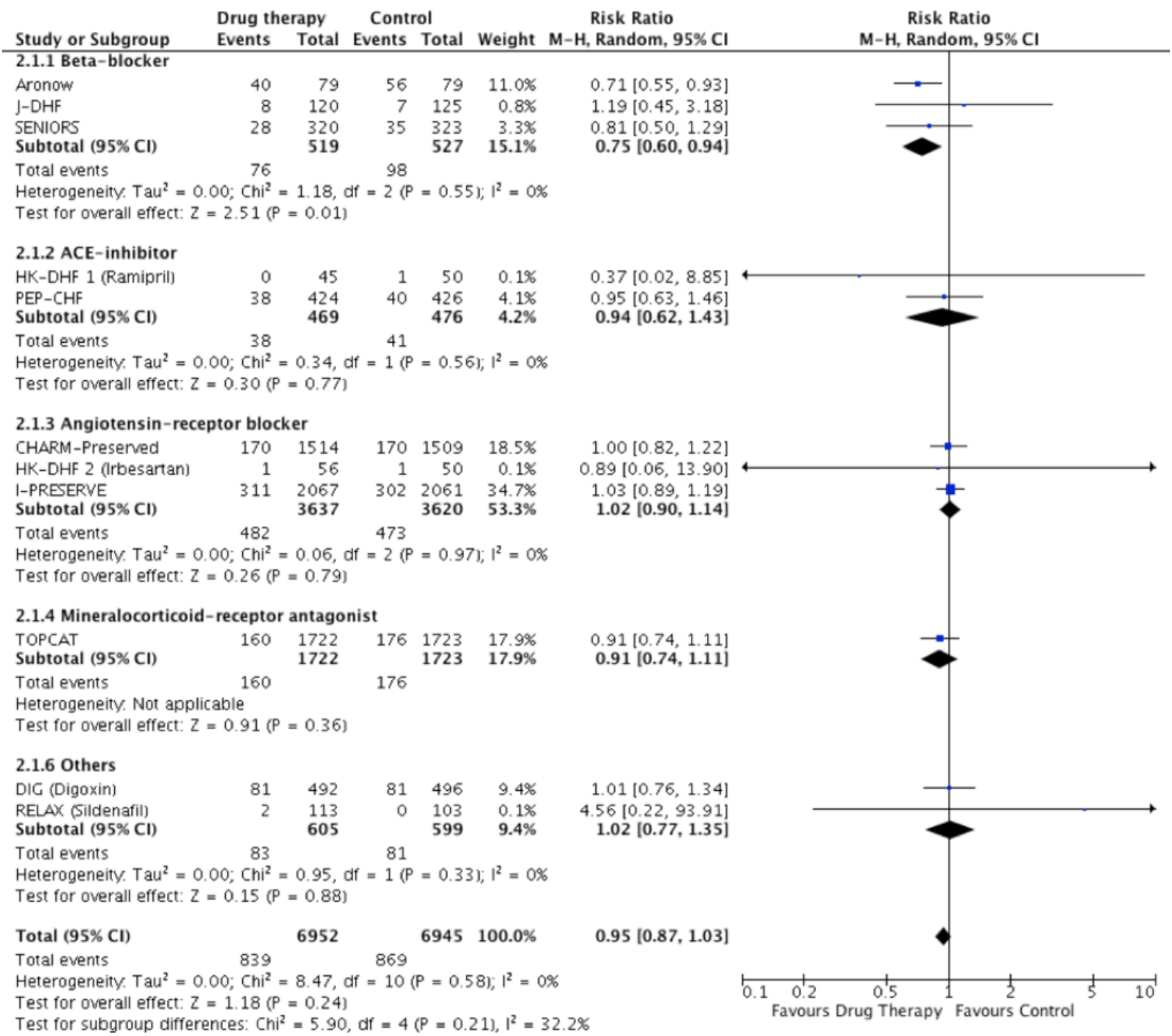

Figure 3 Pooled and individual estimates of relative risk (RR) and $95 \% \mathrm{Cls}$ of the secondary outcome cardiovascular mortality for different therapies. Data are shown stratified by individual class blockers (beta-blockers, ACE inhibitors, angiotensin receptor blockers, mineralocorticoid receptor antagonists and other drug classes). Random-effects model used.

outcomes (all-cause mortality: RR: $0.79,95 \% \mathrm{CI}: 0.66$ to 0.95 ; $\mathrm{p}=0.01$, cardiovascular mortality: RR: $0.71,95 \% \mathrm{CI}: 0.55$ to $0.90, p=0.005)$ compared with those with longer follow-up (>12 months) (all-cause mortality: RR: 0.99, 95\% CI: 0.92 to 1.06, $\mathrm{p}=0.77$; cardiovascular mortality: RR: 0.99 , 95\% CI: 0.90 to $1.08, p=0.76$ ) (see online supplementary figures $1 \mathrm{D}$ and $2 \mathrm{D}$, table 1). Pharmacotherapy reduced heart failure hospitalisations in both shorter and longer trials, with a numerically greater reduction in risk in shorter trials: RR: $0.67,95 \% \mathrm{CI}$ : 0.48 to $0.94, \mathrm{p}=0.02$ and RR: $0.90,95 \% \mathrm{CI}: 0.82$ to 0.98 , $\mathrm{p}=0.02$, respectively $\left(\chi^{2}=2.70, \mathrm{p}=0.10\right)$ (online supplementary figure $3 \mathrm{D})$.

\section{Effect of therapy on exercise capacity}

Ten studies ( $\mathrm{n}=1870$ patients) reported on $6 \mathrm{MWD}$, eight studies $(n=938)$ reported on exercise time after treatment and six studies $(n=924)$ reported on $\mathrm{VO}_{2}$ max. There was no significant difference between groups for exercise time, $\mathrm{VO}_{2}$ max and 6MWD (see online supplementary figure 4).

\section{Effect of therapy on quality of life}

Nine trials reported the treatment effects on quality of life as measured by the MLHFQ, including a total of 3510 patients (beta-blocker: 116 patients, ACE inhibitor: 166, ARB: 2460, MRA: 444, other: 324). Overall estimate showed that treatment resulted in better quality of life scores (MD: $-1.63,95 \% \mathrm{CI}$ : -2.94 to $-0.31, \mathrm{p}=0.001$ ) (see online supplementary figure 5).

\section{Effect of therapy on heart failure biomarkers}

Thirteen trials reported BNP and NT-proBNP levels after treatment (see online supplementary table 3). Studies had a high degree of heterogeneity $\left(\mathrm{I}^{2}=84.4 \%\right)$ and were not further analysed.

\section{Study quality and publication bias}

Five studies were identified as high risk of bias using the Cochrane risk of bias tool, and the remainder low risk (see online supplementary materialsaterials). The funnel plot for all-cause mortality, cardiovascular mortality and heart 


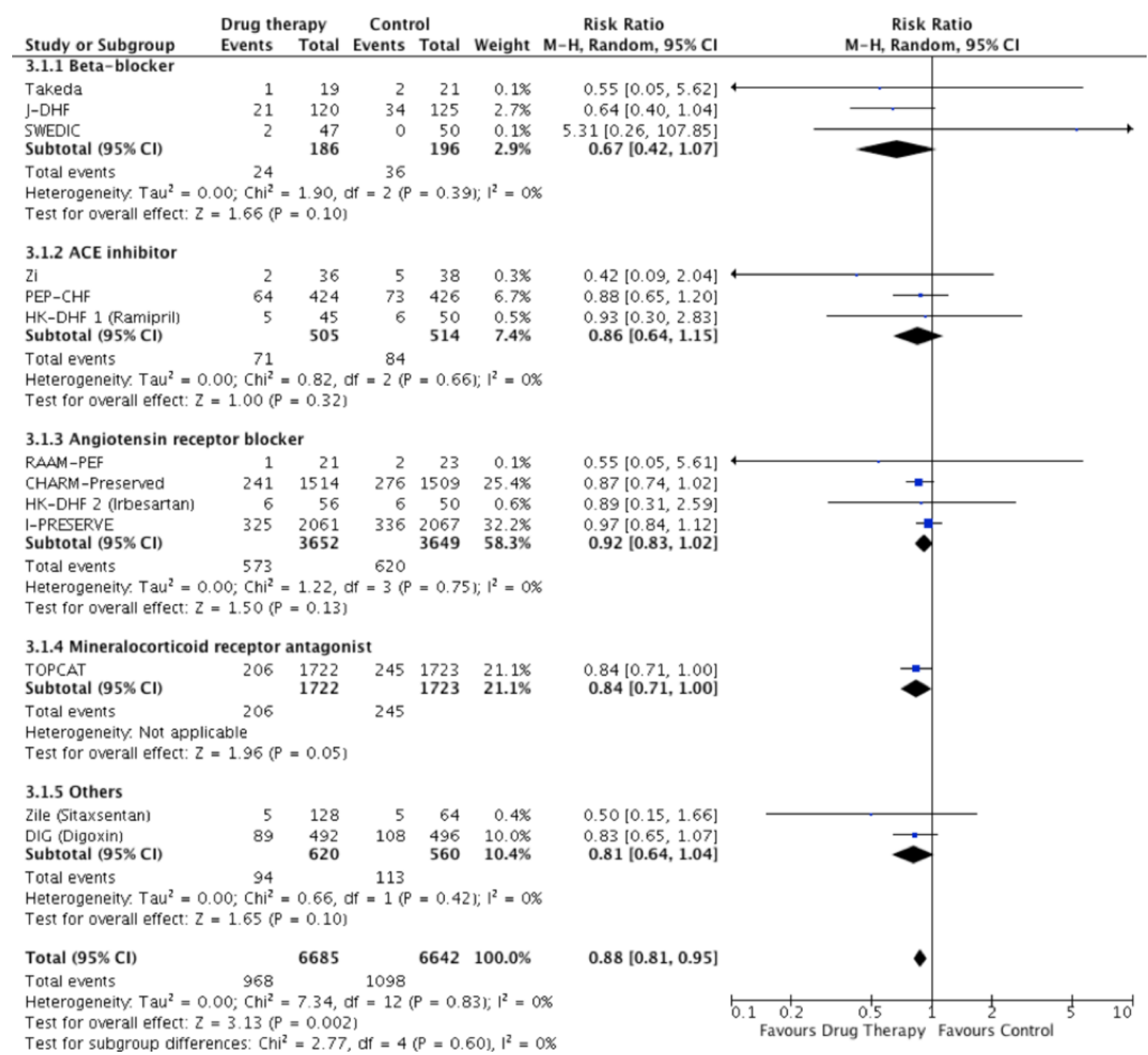

Figure 4 Pooled and individual estimates of relative risk (RR) and $95 \% \mathrm{Cl}$ of the secondary outcome heart failure hospitalisation for different therapies. Data are shown stratified by individual class blockers (beta-blockers, ACE inhibitors, angiotensin receptor blockers, mineralocorticoid receptor antagonists and other drug classes). Random-effects model used.

failure hospitalisation were symmetrical, providing no evidence of publication bias or small study effects (see online supplementary figure 6). Egger test did not identify asymmetry for any of the funnel plots. ${ }^{25}$

\section{DISCUSSION}

The results of this meta-analysis show significant reductions in all-cause and cardiovascular mortality in RCTs using betablockers, while RAAS blockade (using ACE inhibitor, ARB and MRA individually) demonstrated no effect on mortality. Improvements in functional outcomes and quality of life were not significantly or consistently demonstrated using pooled results. Heterogeneity within trials that reported biomarker outcomes was too high to allow comparison.

The effect of beta-blockade on mortality suggests favourable outcomes in patients with LV ejection fraction $>40 \%$. Similar benefits have been demonstrated in pooled analysis of observational studies and previous meta-analyses. ${ }^{26-29}$ Of the three beta-blocker trials, only one trial individually showed a significant reduction in mortality, ${ }^{12}$ and contributed the greatest weight to overall effects due to the high event rate. The two larger RCTs showed neutral results, although were both underpowered to detect effects on mortality. ${ }^{11}{ }^{13}$ Notably, all three beta-blocker trials used an LV ejection fraction threshold of 40\%, whereas trials using ACE inhibitor, ARB and MRA tended to use higher ejection fraction thresholds. The demonstrated reduction in mortality with beta-blockers may have been augmented by their effects on the HFmrEF population within these trials; a group that an emerging body of evidence suggests is more closely aligned with HFrEF. ${ }^{30} 31$ The beneficial effect of betablockers on mortality appears to be through preventing cardiovascular death, supported by a $25 \%$ reduction in cardiovascular mortality. We did not further investigate causes of cardiovascular death, although the pleiotropic effects of beta-blocker (such as its anti-arrhythmic properties) are likely to be important. Future sufficiently powered clinical trials and observational or registry 
Table 1 Summary of effects for all-cause mortality, cardiovascular mortality and heart failure hospitalisation

\begin{tabular}{|c|c|c|c|c|c|c|c|c|c|c|}
\hline \multirow[b]{2}{*}{ Outcome } & \multirow[b]{2}{*}{ All trials } & \multicolumn{3}{|c|}{ Drug classes } & \multicolumn{2}{|c|}{ Follow-up duration } & \multicolumn{2}{|c|}{$\begin{array}{l}\text { Entry LV ejection fraction } \\
\text { threshold }\end{array}$} & \multicolumn{2}{|c|}{$\begin{array}{l}\text { Mean LV ejection } \\
\text { fraction }\end{array}$} \\
\hline & & $\begin{array}{l}\text { Beta- } \\
\text { blockers }\end{array}$ & $\begin{array}{l}\text { RAAS } \\
\text { antagonists }\end{array}$ & Other & $\begin{array}{l}3- \\
12 \text { months }\end{array}$ & $>12$ months & $40 \%-49 \%$ & $\geq 50 \%$ & $<60 \%$ & $\geq 60 \%$ \\
\hline All-cause mortality & $\begin{array}{l}0.96 \\
(0.90 \text { to } \\
1.03)\end{array}$ & $\begin{array}{l}0.78 \\
(0.65 \text { to } \\
0.94) \\
p=0.008\end{array}$ & $\begin{array}{l}1.00 \\
(0.93 \text { to } 1.08)\end{array}$ & $\begin{array}{l}0.95 \\
(0.78 \text { to } 1.15)\end{array}$ & $\begin{array}{l}0.79 \\
(0.66 \text { to } \\
0.95) \\
p=0.01\end{array}$ & $\begin{array}{l}0.99 \\
(0.92 \text { to } 1.06)\end{array}$ & $\begin{array}{l}0.96 \\
(0.88 \text { to } 1.03)\end{array}$ & $\begin{array}{l}0.99 \\
(0.74 \text { to } 1.32)\end{array}$ & $\begin{array}{l}0.93 \\
(0.82 \text { to } \\
1.05)\end{array}$ & $\begin{array}{l}1.01 \\
(0.90 \text { to } \\
1.12)\end{array}$ \\
\hline $\begin{array}{l}\text { Cardiovascular } \\
\text { mortality }\end{array}$ & $\begin{array}{l}0.95 \\
(0.87 \text { to } \\
1.03)\end{array}$ & $\begin{array}{l}0.75 \\
(0.60 \text { to } \\
0.94) \\
p=0.01\end{array}$ & $\begin{array}{l}0.99 \\
(0.89 \text { to } 1.09)\end{array}$ & $\begin{array}{l}1.01 \\
(0.76 \text { to } 1.34)\end{array}$ & $\begin{array}{l}0.71 \\
(0.55 \text { to } \\
0.90) \\
p=0.005\end{array}$ & $\begin{array}{l}0.99 \\
(0.90 \text { to } 1.08)\end{array}$ & $\begin{array}{l}0.95 \\
(0.87 \text { to } 1.03)\end{array}$ & - & $\begin{array}{l}0.90 \\
(0.78 \text { to } \\
1.05)\end{array}$ & $\begin{array}{l}1.02 \\
(0.89 \text { to } \\
1.17)\end{array}$ \\
\hline $\begin{array}{l}\text { Heart failure } \\
\text { hospitalisation }\end{array}$ & $\begin{array}{l}0.88 \\
(0.81 \text { to } \\
0.95) \\
p=0.002\end{array}$ & $\begin{array}{l}0.67 \\
(0.42 \text { to } \\
1.07)\end{array}$ & $\begin{array}{l}0.90 \\
(0.82 \text { to } 0.98) \\
p=0.01\end{array}$ & $\begin{array}{l}0.81 \\
(0.64 \text { to } 1.04)\end{array}$ & $\begin{array}{l}0.67 \\
(0.48 \text { to } \\
0.94) \\
p=0.02\end{array}$ & $\begin{array}{l}0.90 \\
(0.82 \text { to } 0.98) \\
p=0.02\end{array}$ & $\begin{array}{l}0.88 \\
(0.82 \text { to } 0.96) \\
p=0.002\end{array}$ & $\begin{array}{l}0.51 \\
\text { (0.18 to } 1.48)\end{array}$ & $\begin{array}{l}0.85 \\
(0.76 \text { to } \\
0.94) \\
p=0.002\end{array}$ & $\begin{array}{l}0.92 \\
(0.82 \text { to } \\
1.04)\end{array}$ \\
\hline
\end{tabular}

Data presented as risk ratios (for all-cause and cardiovascular mortality and hospitalisation outcomes) or mean difference (exercise capacity, 6MWD, VO max and MLHFQ), with $95 \% \mathrm{Cl}$ and $\mathrm{I}^{2}$ statistic. $p$ values included for analyses that reached statistical significance at $\mathrm{p}=0.05$. RAAS blockers include all trials using ACE inhibitors, angiotensin receptor blockers and mineralocorticoid (each class individually had no effect on all-cause mortality, cardiovascular mortality or heart failure hospitalisation). Only one trial that reported cardiovascular mortality had an entry LV ejection fraction $\geq 50 \%$. Only one trial with LV ejection fraction threshold $\geq 50 \%$ reported cardiovascular mortality. 6MWD, 6 min walk distance; LV, left ventricular; MLHFQ, Minnesota Living With Heart Failure Questionnaire; RAAS, renin-angiotensin-aldosterone system.

data will be important in discerning if there is a true effect in patients with heart failure with an LV ejection fraction of $40 \%$ or more, and whether this benefit extends to those with LV ejection fraction $>50 \%$. While we did not demonstrate a benefit for beta-blockers in hospitalisation for heart failure, 'hospitalisation' should not be interpreted in isolation since mortality events will impact future hospitalisation events.

Given that patients with HFpEF tend to be older than their HFrEF counterparts, and are limited by disabling symptoms with poor quality of life, focus of therapy has shifted towards their effects on exercise tolerance and quality of life. This meta-analysis has shown that drug treatment is associated with a trend towards improvement in exercise time and significant although small improvement in quality of life measured using MLHFQ. Previous meta-analyses have been inconsistent in determining whether treatment improves exercise capacity, ${ }^{3} 32$ and the overall effects are unlikely to be clinically significant.

Interestingly, we showed that trials with shorter follow-up (between 3 and 12 months) demonstrated greater reductions in all clinical end points compared with longer trials. The importance of follow-up duration in HFpEF clinical trials is best highlighted by the Perindopril in Elderly People with Chronic Heart Failure (PEP-CHF) study. There was no significant difference in its primary outcome of combined all-cause mortality and heart failure hospitalisation $(\mathrm{p}=0.92)$ after 2.1 years of follow-up; however, a restrictive analysis at 1-year follow-up demonstrated an important effect in favour of perindopril $(p=0.055)$. It is possible that as follow-up duration increases, cross-over of patients to unblinded study therapy (eg, for treatment of comorbidities) dilutes treatment effects and reduces study power to detect statistically meaningful differences. In this cohort of older and more comorbid patients, treatment efficacy could wane with time as patients develop mortality that is unaffected by treatments.

For hard clinical end points, our study did not find any difference when trials were stratified by LV ejection fraction thresholds (including patients with HFmrEF or not) nor by mean trial LV ejection fractions. Stratifying trials in this way results in smaller heterogeneous subgroups, and therefore evidence is not conclusive. The recently proposed HFmrEF category ${ }^{1}$ currently has no evidence-based treatments, and further clinical trials are required to identify whether this group will benefit from existing treatments used in HFrEF. Since it will be unlikely that dedicated RCTs will be undertaken in this group, meta-analysis using individual patient data and post hoc analysis of large trials will be important in shedding light on this less studied group. Indeed, post hoc analysis of Candesartan in Heart failure: Assessment of Reduction in Mortality and morbidity study (CHARM-Preserved) demonstrates a significant $24 \%$ reduction in CV death and time to first heart failure hospitalisation in patients with HFmrEF (HR: 0.76, 95\% upper CI (UCI) 0.96, p=0.02). ${ }^{33}{ }^{34}$ In TOPCAT, the potential efficacy of spironolactone was greatest in patients with LV ejection fractions $<50 \%$ (HR: 0.72 , UCI 1.05 ) compared with $\geq 60 \%$ (HR 0.97, UCI 1.23). ${ }^{35}$

HFpEF is a highly heterogeneous condition, with interactions between comorbidities likely to account for significant cardiovascular and non-cardiovascular mortality and morbidity. ${ }^{45}$ The effect of treatments on specific HFpEF phenotypes may identify subgroups that benefit. Latent class analysis of the I-PRESERVE data set identified a group of metabolic phenotype patients with HFpEF (high prevalence of diabetes, hyperlipidaemia and obesity) that benefited from irbesartan therapy. ${ }^{36}$ Indeed, further understanding of the pathophysiology of HFpEF will help to guide research and development of novel therapies. ${ }^{15} 37$

\section{Limitations}

The aim of the meta-analysis is to determine treatment effects in patients with an LV ejection fraction $\geq 40 \%$. At the time of undertaking the meta-analysis, these patients were defined as HFpEF, and so the nomenclature has been maintained in this article. Subsequently, there has been a change in definition with introduction of the HFmrEF category. As a result, pooled analysis is for patients with an LV ejection fraction $\geq 40 \%$, and not $\geq 50 \%$. None of the trials reported subgroup data on patients with an LV ejection fraction between $40 \%$ and $49 \%$, and therefore we are unable to report on the treatment effect in these patients. Repeating the meta-analysis with individual patient data will provide greater insights into this intermediate group.

Analyses were stratified by drug class (beta-blockers, RAAS antagonists and other classes). The drugs in the other category include a range of vasodilators, calcium channel blockers and 


\section{Key messages}

\section{What is already known on this subject?}

There have been no individual large randomised controlled trials which demonstrate improvements in clinical outcomes for patients with heart failure and preserved ejection fraction. This is represented in the latest European Society of Cardiology Heart Failure guidelines, which advocate treatment of comorbidities and symptoms.

\section{What might this study add?}

This meta-analysis shows that pooled analysis of beta-blocker trials demonstrates reductions in all-cause and cardiovascular mortality by $22 \%$ and $25 \%$, respectively, with no pooled effect seen in ACE inhibitor, angiotensin receptor blocker and mineralocorticoid antagonist trials. There may also be a small benefit in terms of quality of life with several of the drug classes tested, although no difference in functional outcomes.

\section{How might this impact on clinical practice?}

Clinicians should consider using beta-blockers when there is an existing indication for them. There may be benefit in repeating a randomised controlled trial of beta-blockers in heart failure with preserved left ventricular ejection fraction with contemporary patient cohorts.

digoxin and do not exert their effects through the same mechanisms. We have also made aggregate analyses based on all pharmacological agents, which has limitations, although was used in previous HFpEF meta-analyses due to the small number of trials in each individual drug class. ${ }^{3}$ Where data are available, we have sought to draw conclusions from comparison within drug classes to allow meaningful clinical conclusions to be drawn.

The general limitations associated with meta-analyses extends to this current work. We are limited by availability and reporting of data. ${ }^{38}$ Risk ratios were used as only a small number of trials reported HRs, which introduces bias associated with comparing outcomes in trials of different lengths.

\section{Conclusions}

In trials enrolling patients with HFpEF, defined using an LV ejection fraction $\geq 40 \%$, beta-blockers reduce all-cause and cardiovascular mortality by $22 \%$ and $25 \%$, respectively. There was no significant effect of ACE inhibitors, ARB or MRA on the same outcomes. The effect of treatments on functional and quality of life outcomes was limited. Further adequately powered RCTs in beta-blocker therapy in this patient group is warranted to confirm this finding.

Contributors SLZ initiated the project, designed the search strategy, undertook the search, extracted data and assessed risk of bias, analysed the data and drafted the manuscript. FTC undertook the search, extracted data and assessed risk of bias and edited the manuscript. AAN assisted with assessment of trials for inclusion and edited the manuscript. AMS provided guidance on interpretation of results, critically advising important intellectual content and involved in editing the final manuscript. TM provided guidance on interpretation of results and involved in editing the final manuscript. DOO provided guidance on interpretation of results, critically advising important intellectual content and involved in editing the final manuscript. SA provided statistical guidance, advice on search strategy, undertook statistical analysis and edited the manuscript. All authors approve of the final version to be published. and are accountable to all aspects of the work in ensuring that questions related to the accuracy or integrity of any part of the work are appropriately investigated and resolved.

Funding Open access for this article was funded by King's College London. This work was supported in part by the British Heart Foundation (BHF). Dr Ayis was funded by the National Institute for Health Research (NIHR) Biomedical Research Centre based at Guy's and St Thomas' NHS Foundation Trust and King's College
London. The views expressed are those of the author(s) and not necessarily those of the NHS, the NIHR or the Department of Health.

\section{Competing interests None declared.}

Provenance and peer review Not commissioned; externally peer reviewed.

Open Access This is an Open Access article distributed in accordance with the terms of the Creative Commons Attribution (CC BY 4.0) license, which permits others to distribute, remix, adapt and build upon this work, for commercial use, provided the original work is properly cited. See: http://creativecommons.org/licenses/by/4.0/

(c) Article author(s) (or their employer(s) unless otherwise stated in the text of the article) 2018. All rights reserved. No commercial use is permitted unless otherwise expressly granted.

\section{REFERENCES}

1 Ponikowski P, Voors AA, Anker SD, et al. ESC guidelines for the diagnosis and treatment of acute and chronic heart failure: the Task Force for the diagnosis and treatment of acute and chronic heart failure of the european Society of Cardiology (ESC)Developed with the special contribution of the Heart failure association (HFA) of the ESC. European heart journal 2016 (Epub ahead of print).

2 Yancy CW, Jessup M, Bozkurt B, et al. American College of Cardiology FoundationAmerican Heart Association Task Force on Practice Guidelines. 2013 ACCF/AHA guideline for the management of heart failure: a report of the American College of Cardiology Foundation/American Heart Association Task Force on Practice Guidelines. J Am Coll Cardiol 2013;62:e147-239.

3 Holland DJ, Kumbhani DJ, Ahmed SH, et al. Effects of treatment on exercise tolerance, cardiac function, and mortality in heart failure with preserved ejection fraction. A meta-analysis. J Am Coll Cardio/ 2011;57:1676-86.

4 Mentz RJ, Kelly JP, von Lueder TG, et al. Noncardiac comorbidities in heart failure with reduced versus preserved ejection fraction. J Am Coll Cardiol 2014;64:2281-93.

5 Senni M, Paulus WJ, Gavazzi A, et al. New strategies for heart failure with preserved ejection fraction: the importance of targeted therapies for heart failure phenotypes. Eur Heart J 2014;35:2797-815.

6 Moher D, Liberati A, Tetzlaff J, et al. Preferred reporting items for systematic reviews and meta-analyses: the PRISMA statement. BMJ 2009;339:b2535.

7 DerSimonian R, Kacker R. Random-effects model for meta-analysis of clinical trials: an update. Contemp Clin Trials 2007;28:105-14.

8 Kiran A, Crespillo AP, Rahimi K. Graphics and statistics for Cardiology: data visualisation for meta-analysis. Heart 2017;103:19-23.

9 Higgins JP, Thompson SG, Deeks JJ, et al. Measuring inconsistency in meta-analyses. BMJ 2003;327:557-60.

10 Hand DJ. Measuring classifier performance: a coherent alternative to the area under the ROC curve. Mach Learn 2009;77:103-23.

11 van Veldhuisen DJ, Cohen-Solal A, Böhm M, et al. Beta-blockade with nebivolol in elderly heart failure patients with impaired and preserved left ventricula ejection fraction: data from SENIORS (Study of effects of Nebivolol intervention on outcomes and rehospitalization in seniors with Heart failure). J Am Coll Cardiol 2009;53:2150-8

12 Aronow WS, Ahn C, Kronzon I. Effect of propranolol versus no propranolol on total mortality plus nonfatal myocardial infarction in older patients with prior myocardial infarction, congestive heart failure, and left ventricular ejection fraction $>$ or $=40 \%$ treated with diuretics plus angiotensin-converting enzyme inhibitors. Am J Cardiol 1997:80:207-9.

13 Yamamoto K, Origasa H, Hori M; on behalf of the J-DHF Investigators. Effects of carvedilol on heart failure with preserved ejection fraction: the japanese diastolic Heart failure study (J-DHF). Eur J Heart Fail 2013;15:110-8 http://onlinelibrary.wiley. com/o/cochrane/clcentral/articles/537/CN-00859537/frame.html http://onlinelibrar y.wiley.com/store/10.1093/eurjhf/hfs141/asset/ejhfhfs141.pdf?v=1\&t=iefjj60q\&s= 05fb43d282267b248efddc37e10927936f94060d

14 Yip GW, Wang M, Wang T, et al. The Hong Kong diastolic heart failure study: a randomised controlled trial of diuretics, irbesartan and ramipril on quality of life, exercise capacity, left ventricular global and regional function in heart failure with a normal ejection fraction. Heart 2008;94(5 http://onlinelibrary.wiley.com/o/cochrane/ clcentral/articles/478/CN-00629478/frame.html http://heart.bmj.com/content/94/5/ 573.full.pdf

15 Cleland JGF, et al. The perindopril in elderly people with chronic heart failure (PEPCHF) study. Eur Heart J 2006;27:2338-45 http://onlinelibrary.wiley.com/o/cochrane/ clcentral/articles/489/CN-00576489/frame.html http://eurheartj.oxfordjournals.org/ content/ehj/27/19/2338.full.pdf

16 Davis BR, Kostis JB, Simpson LM, et al. Heart failure with preserved and reduced left ventricular ejection fraction in the antihypertensive and lipid-lowering treatment to prevent heart attack trial. Circulation 2008;118:2259-67.

17 Zi M, Carmichael N, Lye M. The effect of quinapril on functional status of elderly patients with diastolic heart failure. Cardiovasc Drugs Ther 2003;17:133-9 http:// onlinelibrary. wiley.com/o/cochrane/clcentral/articles/791/CN-00457791/frame.html

18 Yusuf S, Pfeffer MA, Swedberg K, et al. Effects of candesartan in patients with chronic heart failure and preserved left-ventricular ejection fraction: the CHARM-Preserved 
trial. Lancet 2003;362:777-81 http://onlinelibrary.wiley.com/o/cochrane/clcentral/ articles/464/CN-00440464/frame.html http://www.thelancet.com/journals/lancet/ article/PIIS0140-6736(03)14285-7/abstract.

19 Solomon SD, Wang D, Finn P, et al. Effect of candesartan on cause-specific mortality in heart failure patients: the Candesartan in Heart failure Assessment of Reduction in Mortality and morbidity (CHARM) program. Circulation 2004;110:2180-3.

20 Massie BM, Carson PE, McMurray JJ, et al. Irbesartan in patients with heart failure and preserved ejection fraction. N Eng/ J Med 2008:359:2456-67.

21 Pitt B, Pfeffer MA, Assmann SF, et al. TOPCAT Investigators. Spironolactone for heart failure with preserved ejection fraction. N Eng/ J Med 2014;370:1383-92 http:// onlinelibrary.wiley.com/o/cochrane/clcentral/articles/299/CN-00989299/frame.htm

22 Edelmann F, Wachter R, Schmidt AG, et al. Effect of spironolactone on diastolic function and exercise capacity in patients with heart failure with preserved ejection fraction: the Aldo-DHF randomized controlled trial. JAMA 2013;309:781-91.

23 Ahmed A, Rich MW, Love TE, et al. Digoxin and reduction in mortality and hospitalization in heart failure: a comprehensive post hoc analysis of the DIG trial. Eur Heart J 2006;27 http:// onlinelibrary.wiley.com/o/cochrane/clcentral//articles/684/CN-00561684/frame.html http:// eurheartj.oxfordjournals.org/content/ehj/27/2/178.full.pdf

24 Redfield MM, Chen HH, Borlaug BA, et al. Effect of phosphodiesterase-5 inhibition on exercise capacity and clinical status in heart failure with preserved ejection fraction: a randomized clinical trial. JAMA 2013;309:1268-77.

25 Egger M, Davey Smith G, Schneider M, et al. Bias in meta-analysis detected by a simple, graphical test. BMJ 1997;315:629-34.

26 Liu F, Chen Y, Feng X, et al. Effects of beta-blockers on heart failure with preserved ejection fraction: a meta-analysis. PLoS One 2014;9:e90555.

27 Bavishi C, Chatterjee S, Ather S, et al. Beta-blockers in heart failure with preserved ejection fraction: a meta-analysis. Heart Fail Rev 2015;20:193-201.

28 Ruiz G, Andrey JL, Puerto JL, et al. Prognosis of heart failure with preserved ejection fraction treated with $\beta$-blockers: a propensity matched study in the community. Int $J$ Cardiol 2016;222:594-602.
29 Lund LH, Benson L, Dahlström U, et al. Association between use of $\beta$-blockers and outcomes in patients with heart failure and preserved ejection fraction. JAMA 2014;312:2008-18.

30 Chioncel O, Lainscak M, Seferovic PM, et al. Epidemiology and one-year outcomes in patients with chronic heart failure and preserved, mid-range and reduced ejection fraction: an analysis of the ESC Heart Failure Long-Term Registry. Eur I Heart Fail 2017 (Epub ahead of print).

31 Lam CS, Solomon SD. Fussing over the Middle Child: heart failure with Mid-Range Ejection Fraction. Circulation 2017;135:1279-80.

32 Fukuta H, Goto T, Wakami K, et al. Effects of drug and exercise intervention on functional capacity and quality of life in heart failure with preserved ejection fraction: a meta-analysis of randomized controlled trials. Eur J Prev Cardiol 2016;23:78-85.

33 PACE. 2017; http://pace-cme.org/2017/04/30/phenotype-hf-with-midrange-efresembles-hfref-albeit-with-better-outcomes/

34 ESC. 2017; https://www.escardio.org/Congresses-\&-Events/Heart-Failure/Congressresources/candesartan-provides-similar-benefit-for-patients-with-mid-range-ejectionfracti/

35 Solomon SD, Claggett B, Lewis EF, et al. Influence of ejection fraction on outcomes and efficacy of spironolactone in patients with heart failure with preserved ejection fraction. Eur Heart J 2016;37:455-62.

36 Kao DP, Lewsey JD, Anand IS, et al. Characterization of subgroups of heart failure patients with preserved ejection fraction with possible implications for prognosis and treatment response. Eur J Heart Fail 2015;17:925-35.

37 Nabeebaccus A, Zheng S, Shah AM. Heart failure-potential new targets for therapy. $\mathrm{Br}$ Med Bull 2016;119:99-110.

38 Zheng SL, Chan FT, Maclean E, et al. Reporting trends of randomised controlled trials in heart failure with preserved ejection fraction: a systematic review. Open Heart 2016;3:e000449 